\title{
BMJ Open Resilience in Healthcare (RiH): a longitudinal research programme protocol
}

\author{
Karina Aase (D) , ${ }^{1}$ Veslemøy Guise (D) , ${ }^{1}$ Stephen Billett, ${ }^{2}$ \\ Stephen Johan Mikal Sollid, ${ }^{1,3}$ Ove Njå, ${ }^{4}$ Olav Røise (1) ,1,5 Tanja Manser, ${ }^{6}$ \\ Janet E Anderson, ${ }^{1,7}$ Siri Wiig ${ }^{1}$
}

To cite: Aase K, Guise V, Billett S, et al. Resilience in Healthcare (RiH): a longitudinal research programme protocol. BMJ Open 2020;10:e038779. doi:10.1136/ bmjopen-2020-038779

- Prepublication history and additional material for this paper are available online. To view these files, please visit the journal online (http://dx.doi. org/10.1136/bmjopen-2020038779).

Received 24 March 2020 Revised 13 August 2020 Accepted 01 October 2020
Check for updates

(C) Author(s) (or their employer(s)) 2020. Re-use permitted under CC BY-NC. No commercial re-use. See rights and permissions. Published by BMJ.

For numbered affiliations see end of article.

Correspondence to

Dr Karina Aase;

karina.aase@uis.no

\section{ABSTRACT}

Introduction Over the past three decades, extensive research has been undertaken to understand the elements of what constitutes high quality in healthcare. Yet, much of this research has been conducted on individual elements and their specific challenges. Hence, goals other than understanding the complex of factors and elements that comprises quality in healthcare have been privileged. This lack of progress has led to the conclusion that existing approaches to research are not able to address the inherent complexity of healthcare systems as characterised by a significant degree of performance variability within and across system levels, and what makes them resilient. A shift is, therefore, necessary in such approaches. Resilience in Healthcare (RiH) adopts an approach comprising a comprehensive research programme that models the capacity of healthcare systems and stakeholders to adapt to changes, variations and/or disruptions: that is, resilience. As such, RiH offers a fresh approach capable of capturing and illuminating the complexity of healthcare and how high-quality care can be understood and advanced.

Methods and analysis Methodologically, to illuminate what constitutes quality in healthcare, it is necessary to go beyond single-site, case-based studies. Instead, there is a need to engage in multi-site, cross-national studies and engage in long-term multidisciplinary collaboration between national and international researchers interacting with multiple healthcare stakeholders. By adopting such processes, multiple partners and a multidisciplinary orientation, the 5-year $\mathrm{RiH}$ research programme aims to confront these challenges and accelerate current understandings about and approaches to researching healthcare quality.

The RiH research programme adopts a longitudinal collaborative interactive design to capture and illuminate resilience as part of healthcare quality in different healthcare settings in Norway and in five other countries. It combines a meta-analysis of detailed empirical research in Norway with cross-country comparison from Australia, Japan, Netherlands, Switzerland and the UK. Through establishing an RiH framework, the programme will identify processes with outcomes that aim to capture how high-quality healthcare provisions are achieved. A collaborative learning framework centred on engagement aims to systematically translate research findings into practice through co-construction processes with partners and stakeholders.
Strengths and limitations of this study

- Moving beyond the individual case study approach and taking a longitudinal, multilevel, cross-case approach to explore the complexities of resilient capacities required for high quality healthcare.

- The 5-year longitudinal research programme offering an integration of resilience theory, collaborative learning as well as patient and stakeholder involvement (PSI) is enacted through a multidisciplinary approach.

- Combining detailed empirical research in diverse healthcare settings in one country, with crosscountry comparison of resilient capacities in six other countries as a basis for meta-analysis.

- A potential limitation is that the programme duration of 5 years may not be sufficient to demonstrate how resilient capacities can improve healthcare quality by means of PSI and collaborative learning.

Ethics and dissemination The $\mathrm{RiH}$ research programme is approved by the Norwegian Centre for Research Data (No. 864334). The empirical projects selected for inclusion in this longitudinal research programme have been approved by the Norwegian Centre for Research Data or the Regional Committees for Medical and Health Research Ethics. The RiH research programme has an embedded publication and dissemination strategy focusing on the progressive sharing of scientific knowledge, information and results, and on engaging with the public, including relevant patient and stakeholder representatives. The findings will be disseminated through scientific articles, $\mathrm{PhD}$ dissertations, presentations at national and international conferences, and through social media, newsletters and the popular media.

\section{INTRODUCTION}

Resilience in healthcare

Resilience in healthcare ( $\mathrm{RiH})$ is central to what constitutes quality in healthcare provision. Defined by the proactive capacity that organisations, units, teams and individuals enact to adapt to changes and potential challenges in everyday practices, rather than to 
resist them, resilience results in high quality care. This way of defining resilience is held to be comprehensible regardless of the healthcare system component or level under investigation. Resilience should, furthermore, be explored as a multilevel phenomenon with collaborative learning and stakeholder involvement as vital prerequisite pillars. ${ }^{1}$ Stakeholders in healthcare are any person, group or organisation who provides, receives, manages, regulates or pays for healthcare. As such, they involve patients, carers, healthcare professionals, managers, regulatory bodies, non-governmental organisations, municipalities, regional authorities and so on.

Resilient healthcare is assumed to be underpinned by adaptive capacity in the healthcare system. It involves the use of internal (eg, sense making, experience) and external resources (eg, colleagues, networks, regulation) to adapt everyday functioning (eg, adaptation of care processes as a result of variability in demand or time constraints), to successfully resolve challenging issues to continue to operate with a high level of quality. ${ }^{2}$ In contrast to much existing research on healthcare quality, which tends to focus on healthcare failures, ${ }^{3}{ }^{4}$ resilience research is focused on capturing healthcare processes with successful outcomes to illuminate how high quality is generated across healthcare systems, organisations and, crucially, in everyday clinical work. ${ }^{5}$ Evidence reported in sectors other than healthcare indicates that resilience comprises capacities such as flexibility, adjustments, improvisation, adaptation and responding to variability. ${ }^{6}$ These capacities are currently explored and partially acknowledged in the healthcare sector, but have to date been limited to small-scale individual case studies, with a few notable exceptions. $^{78}$ Detailed, multi-site, multidisciplinary and multilevel research is, therefore, needed to study $\mathrm{RiH}$ over time and empirically in different clinical and system contexts.

$\mathrm{RiH}$ as conceptualised above is rooted in resilience theory. Resilience is primarily a guiding concept represented in different ways in theories from diverse scientific disciplines. ${ }^{1}$ Engineering and human resources perspectives seek to understand and strengthen how people adapt and build adaptive capacity into technological systems or organisations. Psychological perspectives focus on individual psychological capacities to cope with adversity and is often linked to vulnerable groups. Ecological perspectives focus on how biological systems facing unpredictable changes adapt to cope with these and maintain system stability. Societal perspectives seek to understand and plan responses to and recovery from large scale disasters to preserve system stability and infrastructure. These diverse theories and models about adapting to problems, changes and adversity have informed health services research, including resilient healthcare. As such, RiH is a growing research field that seeks to understand and improve system functioning from institutional, work systems and personal perspectives to deliver high quality care and safe patient care. ${ }^{1}$

\section{Background and status of knowledge}

Healthcare quality is a highly prioritised global health issue that involves the components of patient safety, continuity of care, patient-centredness, effectiveness, equity and efficiency. ${ }^{9}$ Over the past three decades, extensive research has been undertaken to understand these quality components individually. However, many and perhaps most efforts are conducted in silos (ie, mono-disciplinary, without crossing healthcare levels or organisational boundaries) and significant advancements have yet to be made. For example, surveys of patient experiences indicate that hospitals continue to score poorly regarding coordination, continuity of care and patient-centredness. ${ }^{10}{ }^{11}$ Furthermore, data consistently show that, internationally, the rate of harm due to healthcare-induced adverse events remains between 5\% and $10 \%$ for hospitalised patients with some variation across countries. ${ }^{12-16}$ Higher numbers are indicated for primary care patients ${ }^{17}$ including, for example, medication administration-related adverse events at a range of $13 \%-31 \% .^{18}$

The relative consistency of these findings over time and in different geographical locations leads to the conclusion that many existing approaches to research that rely on a range of standardised methods (eg, root cause analysis, checklists, handover protocols) are inadequate for understanding, facilitating and maintaining healthcare quality. Instead, poor healthcare quality is often related to the inherent complexity of healthcare systems, characterised by silos, multiple stakeholder interactions and a significant degree of performance variability within and across system levels. ${ }^{341920}$ A radical change is, therefore, necessary to understand the complexity of care processes, identify how resilience can be supported, and build an interdisciplinary theoretical and methodological approach that bridges the social sciences, the clinical field, and quality and safety research. ${ }^{21}$

Empirical research from across multiple healthcare settings is necessary to capture, illuminate, and, subsequently, support resilient capacities and processes across entire healthcare systems ${ }^{20}$ (eg, by informing the development of interventions to improve capacity for adaptive change). ${ }^{22}$ However, apart from a small number of studies within specific clinical areas, ${ }^{23}$ healthcare research conducted from a resilience perspective is still in its infancy. ${ }^{24}$ The current studies predominantly use qualitative designs ${ }^{232526}$ while a few quantitative and multisite studies exist. ${ }^{7827}$ Therefore, limited understandings are currently available to elucidate how resilience is manifest in different healthcare settings and how factors such as type and pace of clinical work or patient acuity affects the levels of resilience afforded by the healthcare system. Consequently, research is needed to systematically identify and subsequently test and evaluate concepts and interventions that promote resilience as a means to secure high quality healthcare in a variety of healthcare settings and contexts. ${ }^{20}$ 


\section{Research challenges}

The $\mathrm{RiH}$ research programme aims to address six major research challenges related to resilience and its role in high quality healthcare. These are related to the current gaps in the literature concerning:

1. A comprehensive explanatory theory of resilience. Existing efforts to develop sound concepts and models of resilience lack extensive empirical testing. ${ }^{28-30}$ Existing research also lacks theoretical integration of the multiple levels of the healthcare system, from individuals and teams (micro), to organisations (meso), to regulatory bodies and policy level actors (macro).

2. Patient and stakeholder involvement (PSI) in studies of $\mathrm{RiH},{ }^{31}$ despite persistent claims within the literature that patients, carers and other healthcare stakeholders are fundamental cocreators of resilience. ${ }^{3} 20$ 32-34 Addressing this shortcoming contains the need to develop appropriate procedures for PSI throughout the research process ${ }^{35}$ which includes investigating how patients can contribute to resilience.

3. Comprehensive methodological approaches to improve the validity, reliability and efficiency of RiH research. Innovative methods are required to identify and document adaptive capacities in a longitudinal perspective. $^{22}{ }^{23}$ Systematic use of multi-method and meta-synthesis approaches are needed to secure comprehensive understandings of resilient capacities and processes. ${ }^{6}$

4. Multilevel, multi-context and cross-country resilience studies. There is a need for a broad, interlinked set of empirical studies to identify and describe resilience at multiple levels of the healthcare system, in different empirical settings, and in cross-country comparative studies. ${ }^{635}$ To date, most resilience studies have been conducted in acute/emergency room settings. ${ }^{23}$ The role of various contextual factors (eg, regulatory systems, cultural and organisational factors, leadership) remains relatively unexplored and in need of investigation and theorising. ${ }^{30} 36$

5. Validated indicators and outcome measures to assess resilience in high quality healthcare settings. In conducting comprehensive analyses of how systems become resilient, validated measures for detecting, measuring, assessing and verifying adaptive capacities are urgently needed. ${ }^{622}$

6. Evidence-based interventions and improvement efforts. Large-scale research studies are needed to identify, develop and evaluate RiH interventions that enable integration into improvement efforts and management routines in practice. ${ }^{6}$

This longitudinal research programme will address these challenges by bringing quality and safety expertise together with healthcare professionals, patients and other stakeholders, providing a unique opportunity to conduct comprehensive multidisciplinary research that goes beyond current practices in five distinct ways: First, by developing an RiH framework based on resilience as originally applied in psychology, ecology and engineering ${ }^{1}$; second, by using a transdisciplinary, multilevel (micro/ meso/macro) approach ${ }^{23}$; ; third, by synthesising longitudinal in-depth studies in differing empirical healthcare settings to identify context specific and/or context independent features of resilience; fourth, by conducting cross-country comparative studies; and fifth, by focusing on the role that patients and other stakeholders have in $\mathrm{RiH}$ research and practice.

\section{Objective and research questions}

The primary objective of the research programme, it follows, is to reform the understanding of quality in healthcare by the development, implementation, and test of a theoretical and practical RiH framework. The RiH programme covers the quality components of patient safety, continuity of care, patient-centredness and clinical effectiveness. More specifically, the $\mathrm{RiH}$ programme addresses the following research questions:

1. How can an integrative theoretical framework for RiH be described to understand and improve quality at different system levels?

2. How can involvement of patients and stakeholders in RiH be described and improved?

3. How can RiH be described and improved in different healthcare settings?

4. How can the role of collaborative learning in $\mathrm{RiH}$ be described and improved?

5. How can RiH be identified, analysed and compared in different international healthcare settings?

\section{Research setting}

The RiH programme is primarily centred around the Norwegian healthcare system. Through a cross-country comparative study, the international healthcare context is included covering five other countries (Australia, England, Japan, Netherlands and Switzerland). These countries are strategically selected to represent a variety of developed healthcare systems and demographics. The international research setting is described in more detail in a forthcoming study protocol focusing on the crosscountry comparison. ${ }^{38}$

As in most other developed countries, the focus on healthcare quality and safety in Norway has increased over the past 15 years. The first national patient safety campaign (2010-2013) initiated a systematic measurement of patient harm in Norwegian hospitals. The number of harmed hospitalised patients was stable at around 14\% from 2012 to 2017, with a significant reduction in 2018 to $11.9 \%$. There has been a major focus on establishing stable structures and cultures for patient safety in the specialised healthcare services. The National Patient Safety programme (2014-2018) continued the attention on quality and safety and was supported by a leadership-focused regulatory effort (2017) pointing to the role of managers in primary and specialised healthcare services in ensuring high quality services. Since 2013, Norway has published annual reports to the Parliament (Storting) on the status, challenges and measures for 
quality and safety in healthcare. In 2019, the Norwegian Directorate of Health launched a new 5-year action plan for patient safety and quality improvement (2019-2023). It states that despite the implementation of national quality indicators, quality registries, patient pathways, patient harm measurements, electronic patient records, infection prevention and improvement bundles, there are still challenges in need of a national and coordinated effort. $^{39}$

The Norwegian population is 5.3 million and the healthcare system is semi-decentralised mainly based on public funding. The state is responsible for specialist care (administered by four Regional Health Authorities) and the municipalities are responsible for providing primary care services to its inhabitants (eg, nursing homes, home care, general practitioners, emergency clinics). The country is characterised by large geographical distances with variation across counties from the most central parts around the largest cities, to the most rural areas in the northern part of the country. Some counties are characterised by fjords, mountains, a harsh and dark winter climate, and long distances to the nearest hospitals, including ferries from islands or across fjords. Seventy-five per cent of the population live in the 100 most densely populated municipalities. The most highly populated municipality has 673000 inhabitants, while the smallest has 200 inhabitants. $^{40}$

The regulation of quality and safety in healthcare, like in many other sectors in Norway, is based on enforced self-regulation and internal control. ${ }^{41}$ The Norwegian Board of Health Supervision is the national regulatory body for health and care services administered by the Ministry of Health and Care Services. At the regional level, 11 county governors oversee and inspect services within both primary and specialised healthcare. The 356 municipalities are granted wide discretion in organising of primary care services.

\section{METHODS AND ANALYSIS \\ Overall design}

A collaborative interactive research design will be used to ensure the establishment of a comprehensive RiH framework with both theoretical and practical outcomes. This type of collaborative design is ideal when the aim is to bring key actors (multidisciplinary researchers, practitioners, patients and other stakeholders) together in a partnership centred on undertaking multiple phases of development, implementation, evaluation and improvement ${ }^{42-44}$ as is the purpose of the $\mathrm{RiH}$ research programme.

Iterative cycles of research activities will be organised in five closely integrated work packages (WPs) (see figure 1). An integrative theoretical framework will be established (WP1), alongside a conceptual model for PSI in RiH (WP2) and best collaborative learning methods to translate the RiH framework into practice (WP4). Synthesising findings across in-depth longitudinal empirical research in a sample of healthcare settings in Norway (WP3) and in a cross-country comparative study (WP5) will establish resilient capacities facilitating a common inclusive $\mathrm{RiH}$ framework.

The 5-year research programme (1 September 2018 to 31 October 2023) will have two main phases: an explorative phase with screening, synthesis and validation of results from a sample of existing empirical projects in different healthcare settings, and an intervention phase with design, implementation and evaluation of measures to support resilient capacities in healthcare quality.

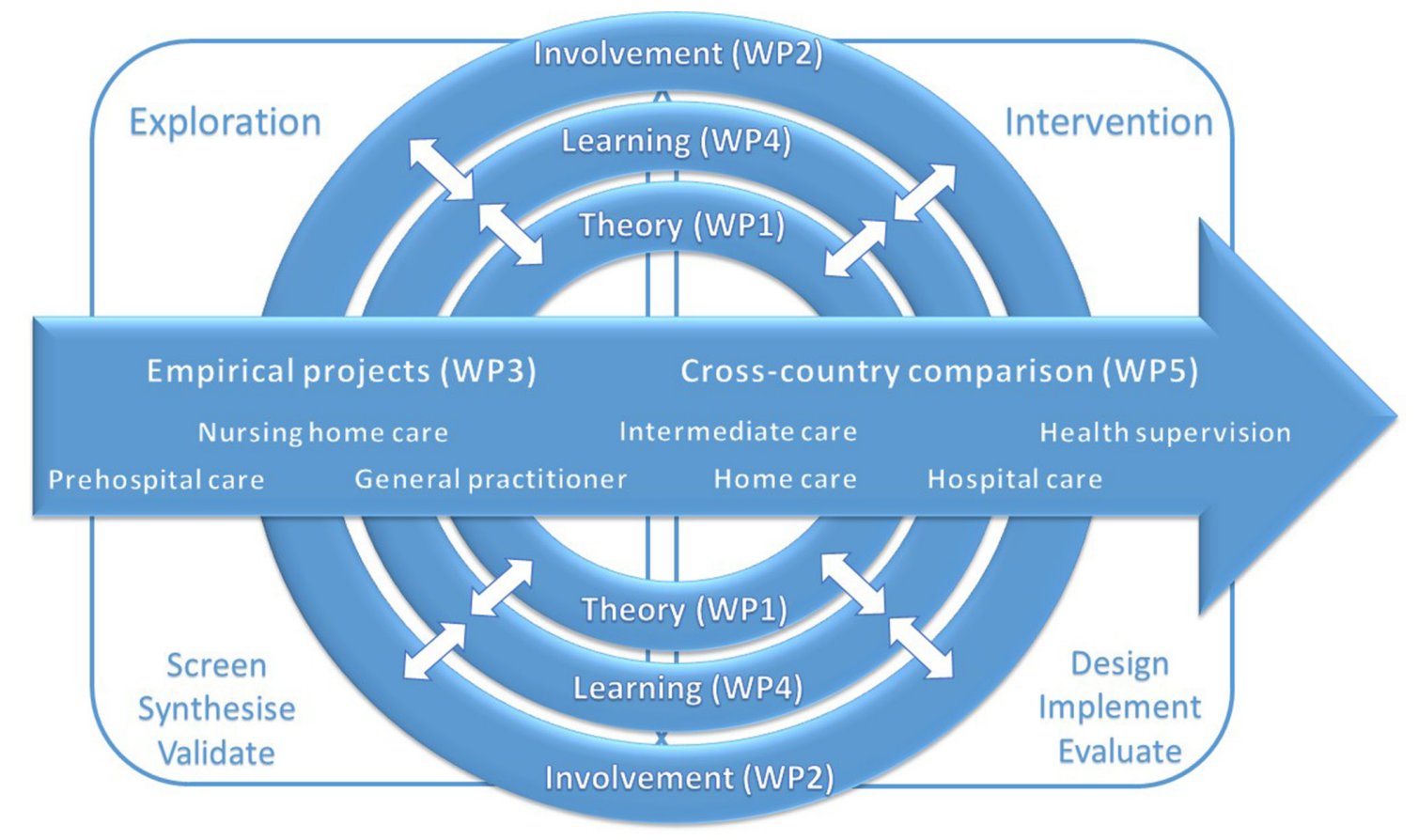

Figure 1 Overview of the resilience in healthcare research programme. 
WPs 1, 2 and 4 constitute the main conceptual WPs in the research programme (see figure 1). These WPs will collect data across different empirical projects in Norway (WP3) and internationally (WP5) and will contribute to WP3 and WP5 by developing theoretical approaches, PSI, and collaborative learning tools in an iterative process. WPs 1-4 will progress across the entire programme period (2018-2023) while WP5 will commence and progress across the second half of the programme period (2020-2023). The three conceptual WPs are also interconnected and relate to each other in different ways. The following relationships will be studied in the $\mathrm{RiH}$ research programme:

- Enactment of adaptive capacity at different levels of the healthcare system requires PSI, and, therefore, there is a need to understand its role in resilience and how it can be developed and supported.

- Enactment of adaptive capacity at different levels of the healthcare system requires collaborative learning and working, and, therefore, there is a need to understand the role of collaborative learning and how it can be developed and supported.

- PSI in the enactment of adaptive capacity requires collaborative learning and working, and, therefore, there is a need to understand, develop, implement and evaluate collaborative learning tools and resources for such activities.

\section{Sample of empirical projects}

The RiH research programme will use data from a broad sample of empirical projects from a wide variety of healthcare settings. The sample will be drawn from a set of former, ongoing or recently granted research projects in which members of the Centre for Resilience in Healthcare in Norway are involved. Approximately 50 research projects, postdoctoral projects and $\mathrm{PhD}$-projects will be screened according to a set screening protocol (see online supplemental file 1) and a Quality and Resilience Trigger Tool (see online supplemental file 2) to establish how they relate to resilience and which healthcare quality components they cover. After screening, a total sample of approximately 20 empirical projects will be selected to secure a comprehensive range of healthcare settings (see figure 1), stakeholders (ie, patients, carers, healthcare professionals, managers, regulators, local and national healthcare authorities), quality dimensions (patient safety, continuity of care, patient-centredness, clinical effectiveness) and adaptive capacities (individual, team/ unit, organisational, larger system).

The sample of empirical projects will be subject to two types of analyses; a broad explorative meta-synthesis of all projects, and an in-depth deductive content analysis of a sub-sample of projects according to categories identified in the explorative synthesis.

For the international cross-country resilience study (WP5), empirical case studies will be conducted in six countries according to an agreed study protocol. ${ }^{38} \mathrm{~A}$ set of case studies from a selection of hospitals in Australia,
England, Japan, Netherlands, Switzerland and Norway will form the international sample.

\section{WP descriptions}

In the following, the five WPs are described with regards to main purpose, research question and work tasks including the activities detailing relationships across WPs.

\section{WP1: RiH theoretical framework}

The purpose of WP1 is to establish a robust, validated, integrative and comprehensive $\mathrm{RiH}$ theoretical framework to improve the understanding of quality in different healthcare settings and at different system levels. The theoretical framework development will include a review of the literature and synthesis of data across the empirical projects included in the $\mathrm{RiH}$ programme. The validation of the framework will occur in selected healthcare settings and through targeted empirical processes including use of PSI and collaborative learning resources.

\section{Research question}

How can an integrative theoretical framework for RiH be described to understand and improve quality at different healthcare system levels?

\section{Work tasks}

1. Develop an initial theoretical framework for resilience by reviewing relevant theoretical concepts, including resilience at an individual psychological level (ie, micro) $)^{45}$; at an organisational level (ie, meso) ${ }^{24-646}$; and at a national and international level (ie, macro). ${ }^{35} 47 \mathrm{~A}$ group of acknowledged expert researchers will attend a workshop to advance and evaluate concepts and principles of resilience and their inter-relations, leading to the development of an initial theoretical framework presented and published in an edited volume.

2. Develop empirical and analytical indicators at the micro, meso and macro levels by positioning the RiH programme understanding of resilience in relation to the existing concepts identified in work task 1 , and by developing empirical indicators for resilience to be used in selected and diverse data collection tools.

3. Collect and synthesise empirical evidence from the selected projects (WP3) in different settings as input to context specific and system level specific elements of the theoretical framework. Empirical evidence will be collected and analysed using a qualitative meta-synthesis approach $^{48}$ on the basis of the sample of empirical projects, meaning that data collection will be conducted across current project documentation (protocols, summaries, publications, etc), researchers involved (interviews, focus groups) and project activities (observations).

4. Validate the integrative RiH framework based on analytical syntheses of empirical evidence collected as part of work task 3 and test the framework in a selected sample of projects (WP3, WP5) across system levels using member checks, group techniques and workshops. Patient and stakeholder analyses conducted in WP2 
and collaborative learning tools developed in WP4 will form the basis for these processes. The validated integrative $\mathrm{RiH}$ framework will include the possibility for creating specific models or resilience representations adjusted to different empirical settings or system levels.

\section{Intended outcomes}

1. Integrative $\mathrm{RiH}$ theoretical framework.

2. Multilevel empirical and analytical resilience indicators.

3. Specific models or representations of resilience in different healthcare settings.

\section{WP2: PSI in RiH}

The purpose of WP2 is to describe how patients and stakeholders are actively involved in creating and sustaining $\mathrm{RiH}$ and how these practices can be supported and improved to facilitate high-quality healthcare. WP2 will employ a collaborative interactive research design involving patients, carers, healthcare professionals, regulatory bodies, the public and other major stakeholders (eg, patient organisations, policy makers) as equal partners at all stages of the research process. ${ }^{44}$

\section{Research question}

How can involvement of patients and stakeholders in $\mathrm{RiH}$ be described and improved?

\section{Work tasks}

1. Establish the current knowledge base on PSI in RiH. A scoping review of the literature will be conducted to establish the current knowledge on patient and stakeholder contributions to RiH. The literature will be analysed according to how and in which healthcare settings different groups of patients and stakeholders contribute to resilience across different system levels.

2. Conduct patient and stakeholder analyses in the core sample of empirical projects. Within a sub-sample of empirical projects included in WP3, we will conduct systematic patient and stakeholder analyses ${ }^{49}$ to generate knowledge about how relevant actors understand and articulate their interests, interrelations, agendas, and the influence or resources they bring to bear on $\mathrm{RiH}$. The stakeholder analyses will form the basis for developing key principles for how the conceptual model for PSI in $\mathrm{RiH}$ can be co-produced and designed.

3. Explore and develop a conceptual model for how PSI in RiH can be understood and improved. A conceptual model for PSI in RiH will be developed by synthesising results on how patients and stakeholders are involved and contribute to $\mathrm{RiH}$ in the sample of empirical projects included in WP3, and how the drivers for their involvement can be understood. Following the patient and stakeholder analyses conducted as part of work task 2, key actors will participate in a co-production process to develop the model by taking roles as panel members, user representatives, advisors or 'co-researchers' (employed in part time posts). WP2 will collect data across the sample of empirical projects using multiple meth- ods (WP3). Topics to be covered are situations where patients and stakeholders contribute to resilience, types of involvement, drivers and barriers to involvement, contextual issues and so on.

4. Evaluate and refine the PSI in RiH model by using collaborative learning tools and innovations. The conceptual model for PSI in RiH will be tested and validated through the development of a set of learning tools and innovations (eg, 'meeting arenas', simulation scenarios) in close collaboration with WP4. The co-production process described in work task 3 will continue to cover these issues.

\section{Intended outcomes}

1. Conceptual model for PSI in RiH.

2. Summary of patient and stakeholder analyses across different healthcare settings.

\section{WP3: RiH in Norwegian healthcare settings}

The purpose of WP3 is to document adaptive capacities across the broad sample of empirical projects included in the research programme, to conduct detailed analyses of selected projects, and based on this develop and implement measures for adaptive capacities in selected healthcare settings by means of PSI and collaborative learning.

\section{Research question}

How can RiH be described and improved in different healthcare settings?

\section{Work tasks}

1. Analyse the broad sample of empirical projects using a qualityresilience framework. For the total sample of empirical projects (approximately 20 projects) a framework for analysis will be developed for establishing the relationship between adaptive capacities and healthcare quality dimensions, based on the Quality and Resilience Trigger Tool (see online supplemental file), system levels, and empirical setting. The analytical framework will be applied on data collected using project documentation (eg, publications, study protocols, reports, etc), semi-structured interviews or focus group interviews with project researchers, or observation of project meetings, workshops and so on.

2. Synthesise in-depth analyses of a sub-sample of empirical projects. For a sub-sample of empirical projects deductive content analyses ${ }^{50}$ will be synthesised across system levels and healthcare settings. The content analyses will be based on categories resulting from the metaanalysis in work task 1. Analytical workshops will be held with the respective project researchers and with patient and stakeholder co-researchers to produce the synthesis. Results will be presented as a set of indicators for adaptive capacities at the micro, meso and macro levels in close collaboration with WP1.

3. Develop, implement and evaluate RiH interventions. Based on the synthesis produced in work task 2 , an $\mathrm{RiH}$ intervention bundle (three to five measures) will be co-produced with the patient and stakeholder groups 
identified in WP2 using the collaborative learning tools established in WP4. The intervention bundle contents will be based on empirical evidence gathered in the analyses of work task 1 and work task 2 and will be implemented in three selected healthcare settings (micro, meso, macro). Possible impact of the RiH intervention bundle will be measured using a process evaluation approach. ${ }^{51}$

\section{Intended outcomes}

1. Analytical framework for establishing the relationship between RiH and quality.

2. Meta-synthesis of adaptive capacities across different healthcare settings.

3. RiH intervention bundle.

\section{WP4: collaborative learning in RiH}

The purpose ofWP4 is to describe the relationship between collaborative learning and resilience to establish a framework that supports adaptive capacities across diverse healthcare settings and system levels. The collaborative learning framework will build on interactive, participatory and reflexive approaches. In developing the framework, adjacent conceptual approaches will be consulted, for example, the relationship-based approach. ${ }^{52}$

\section{Research question}

How can the role of collaborative learning in $\mathrm{RiH}$ be described and improved?

\section{Work tasks}

1. Establish the current knowledge base on collaborative learning in $\mathrm{RiH}$. A review of the literature will be conducted to provide an overview of the role of collaborative learning in current $\mathrm{RiH}$ studies. This literature will be analysed according to how learning is described in relation to resilience, where learning occurs, who is involved in learning situations, and how adaptive capacities are learnt, and that learning is enhanced through collaborative arrangements.

2. Analyse data from the sample of empirical projects in WP3 to identify collaborative learning needs and pedagogically rich activities. Collaborative learning needs and pedagogically rich activities in healthcare settings will be synthesised across the empirical projects included in WP3. WP4 will collect data across the empirical projects using multiple methods (WP3). Processes appraised in these projects will include collaborative meetings, teamwork, learning arenas, learning resources, learning from positive situations, interfaces, participants and so on.

3. Apply participatory design principles to develop and pilottest a set of collaborative learning tools and components $(C L$ framework) to support adaptive capacities. In collaboration with WP2 and based on the learning needs and pedagogically rich activities identified in WP3 analyses, participatory design processes will be run with the aim of developing the contents of, and the underlying principles of a CL framework. The contents of the framework will likely include learning tools such as structured meeting arenas, simulation scenarios, interactive digital guides, webinars, e-dialogue forums and so on. The underlying principles of the framework will address issues such as learning goals, participants, procedures, resources and so on.

4. Implement and evaluate the CL framework in selected empirical settings. The pilot-tested CL framework will be revised and subsequent trials implemented in three different empirical settings in collaboration with WP3; one focusing on healthcare professionals' individual and team-based adaptive capacities (ie, micro level), one focusing on organisational adaptive capacities (ie, meso level) and one focusing on intra-organisational adaptive capacities (ie, macro level). An RiH laboratory will be created to support the implementation. Possible impact of the CL framework will be studied using a participant observation approach ${ }^{53}$ as part of the process evaluation in WP3.

\section{Intended outcomes}

1. Collaborative learning framework for RiH.

2. Synthesis of learning mechanisms for adaptive capacities across different healthcare settings.

\section{WP5: cross-country comparative resilience studies}

The purpose of WP5 is to conduct, analyse and compare empirical studies in different countries to establish the cross-country characteristics of $\mathrm{RiH}$ and to explore under which conditions $\mathrm{RiH}$ is enacted in different countries. The cross-country RiH studies will be conducted in six countries; Netherlands, Australia, England, Switzerland, Japan and Norway according to a joint study protocol. ${ }^{38}$ The international study will follow a three-phased approach including mapping of country characteristics and sampling (phase 1), within country data collection using observations and interviews (phase 2) and crosscountry comparative analysis (phase 3). In phases 2-3 WP5 will focus empirically and analytically on healthcare teams and how they communicate and coordinate to adapt and respond to challenges and problems.

\section{Research question}

How can RiH be identified, analysed and compared in different international settings?

\section{Work tasks}

1. Conduct an initial analysis of the healthcare systems in the six countries. The analysis will be based on mapping and comparison of healthcare contextual issues such as funding and access, patient rights, regulatory framework, accreditation and monitoring, information availability, and resources available.

2. Conduct exploratory empirical case studies of $\mathrm{RiH}$ within selected healthcare settings in the six countries. This will involve interviews at different levels (ie, micro, meso, macro) and observations of clinical work, team and managerial processes in predefined empirical fields. 
The aim will be to identify adaptive capacities across countries, system levels and empirical contexts.

3. Conduct within-case analysis of RiH in each country according to a joint protocol to determine adaptive capacities at micro, meso and macro levels in the selected empirical settings in each country. The 'within-case' analysis will result in six country-specific reports in English to enable cross-country comparison.

4. Conduct cross-case analysis of RiH to synthesise and compare findings across the six country reports using a common conceptual framework. Comparing results across multiple empirical settings, levels and countries will allow identification of differing adaptive capacities and how they are shaped by organisational, cultural, economic and regulatory factors.

5. To develop guidance for policy makers, managers and practitioners for operationalising and implementing $\mathrm{RiH}$ in different countries and organisational contexts. This will include developing freely accessible anonymised RiH case study summaries to inform further development of $\mathrm{RiH}$ studies and interventions, and to influence quality and safety programmes internationally.

\section{Intended outcomes}

1. Summary of healthcare system mapping across six countries.

2. Six country-specific reports on adaptive capacities across system levels.

3. Synthesis of RiH across six countries.

4. RiH guidance for stakeholders in different countries.

\section{Patient and public involvement}

The RiH programme integrates PSI throughout all research phases from project design, planning, data collection, analysis and publication. A specific WP (WP2) is set aside to assure involvement at all levels of the Norwegian healthcare system. A patient and citizen representative has been involved in the programme development and is co-leading the international Expert Advisory Board established in the programme. Co-researchers will be employed in different roles throughout the programme.

\section{ETHICS AND DISSEMINATION \\ Ethics}

The RiH programme is approved by the Norwegian Centre for Research Data (No. 864334). Each individual study in the longitudinal research programme constituting the empirical WP3 will apply for ethical approval from the Norwegian Centre for Research Data or the Regional Committees for Medical and Health Research Ethic. As different countries have different requirements for ethical approval of research studies, each study in the cross-country WP5 will apply for ethical approval in the respective country if required.

\section{Dissemination}

The RiH research programme has a publication and dissemination strategy focusing on the sharing of scientific knowledge, information and results, and on public engagement from relevant patient and stakeholder representatives. The results from the research programme will be disseminated through scientific articles, $\mathrm{PhD}$ dissertations, and presentations at national and international conferences, as well as in social media, newsletters and in the press.

The research programme will organise annual RiH research seminars for academic partners, empirical partners and stakeholders. RiH consortium members, Expert Advisory Board members and leading international researchers will be invited to present stateof-the-art research on RiH. Annual RiH patient/ stakeholder seminars will contribute to create a collaborative learning arena involving relevant stakeholders in the Norwegian healthcare system and $\mathrm{RiH}$ researchers, to enable translation of research evidence into interventions for stakeholder use. The RiH programme will use new forms of virtual platforms to ensure continuous direct communication on a regular basis among the consortium partners. RiH will also take advantage of virtual share-points to enable the secure sharing of documents between partners.

Author affiliations

${ }^{1}$ SHARE - Centre for Resilience in Healthcare, University of Stavanger, Stavanger, Norway

${ }^{2}$ School of Education and Professional Studies, Griffith University, Nathan, Queensland, Australia

${ }^{3}$ Department for Research and Development, Norwegian Air Ambulance Foundation, Oslo, Norway

${ }^{4}$ Faculty of Science and Technology, University of Stavanger, Stavanger, Norway

${ }^{5}$ Division of Orthopedic Surgery, Oslo University Hospital, Oslo, Norway

${ }^{6}$ School of Applied Psychology, University of Applied Sciences and Arts Northwestern Switzerland, Olten, Switzerland

${ }^{7}$ Florence Nightingale Faculty of Nursing, Midwifery \& Palliative Care, King's College London, London, UK

\section{Twitter Olav Røise @0lavroi}

Acknowledgements We thank Roland Bal, Jeffrey Braithwaite, Mathilde Bourrier, Carolyn Canfield Mary Chambers and Marianne Storm for their intellectual inputs to the grant application resulting in the RiH Research Program. We also thank Cecilie Haraldseid-Driftland for comments on an earlier draft of the protocol, and Janne Gro Alsvik for help in preparing the manuscript for submission.

Contributors KA designed the overall research proposal, drafted the original manuscript and contributed to revisions and additions to the manuscript. SW and VG contributed with substantial intellectual contents in the design of the research programme, drafted substantial parts of the original manuscript, and contributed to revision to the manuscript. SJMS, ON, OR, SB, TM and JEA contributed to the design of the research programme, drafted parts of the original manuscript and contributed to revisions to the manuscript. All authors approved the final version of the manuscript.

Funding The RiH research programme has received funding from the Research Council of Norway under the FRIPRO Toppforsk programme, grant agreement no 275367. The University of Stavanger, Norway, the Norwegian University of Science and Technology in Gjøvik, and the Norwegian Air Ambulance Foundation support the programme with in-kind funding.

Competing interests None declared.

Patient consent for publication Not required. 
Provenance and peer review Not commissioned; externally peer reviewed.

Supplemental material This content has been supplied by the author(s). It has not been vetted by BMJ Publishing Group Limited (BMJ) and may not have been peer-reviewed. Any opinions or recommendations discussed are solely those of the author(s) and are not endorsed by BMJ. BMJ disclaims all liability and responsibility arising from any reliance placed on the content. Where the content includes any translated material, BMJ does not warrant the accuracy and reliability of the translations (including but not limited to local regulations, clinical guidelines, terminology, drug names and drug dosages), and is not responsible for any error and/or omissions arising from translation and adaptation or otherwise.

Open access This is an open access article distributed in accordance with the Creative Commons Attribution Non Commercial (CC BY-NC 4.0) license, which permits others to distribute, remix, adapt, build upon this work non-commercially, and license their derivative works on different terms, provided the original work is properly cited, appropriate credit is given, any changes made indicated, and the use is non-commercial. See: http://creativecommons.org/licenses/by-nc/4.0/.

\section{ORCID iDs}

Karina Aase http://orcid.org/0000-0002-5363-5152

Veslemøy Guise http://orcid.org/0000-0002-9124-1664

Olav Røise http://orcid.org/0000-0003-4931-7001

\section{REFERENCES}

1 Wiig S, Aase K, Billett S, et al. Defining the boundaries and operational concepts of resilience in the resilience in healthcare research program. BMC Health Serv Res 2020;20:330.

2 Sutcliffe KM, Vogus TJ. Organizing for resilience. In: Cameron KS, Dutton JE, Quinn RE, eds. Positive organizational scholarship: foundations of a new discipline. San Francisco: Berett-Koehler Publishers, 2003: 94-110.

3 Hollnagel E, Wears RL, Braithwaite J. From Safety-I to Safety-II: A White Paper. The Resilient Health Care Net. University of Southern Denmark, University of Florida, USA, and Macquarie University, Australia, 2015

4 Braithwaite J, Wears RL, Hollnagel E. Resilient health care: turning patient safety on its head. Int J Qual Health Care 2015;27:418-20.

5 Hollnagel E, Woods DD, Leveson N. Resilience engineering: Concepts and precepts. Ashgate: Farnham, 2006.

6 Righi AW, Saurin TA, Wachs P. A systematic literature review of resilience engineering: research areas and a research agenda proposal. Reliab Eng Syst Saf 2015;141:142-52.

7 Gittell JH. Relationships and resilience: care provider responses to pressures from managed care. J Appl Behav Sci 2008;44:25-47.

8 Vogus TJ, lacobucci D. Creating highly reliable health care: how Reliability-Enhancing work practices affect patient safety in hospitals. ILR Review 2016;69:911-38.

9 WHO. Quality of care: A process for making strategic choices in health systems. Geneva: World Health Organization, 2006.

10 Osborn R, Moulds D, Squires D, et al. International survey of older adults finds shortcomings in access, coordination, and patientcentered care. Health Aff 2014;33:2247-55.

11 Holmboe O, ØA B. Pasienterfaringer med norske sykehus i 2014. Lokale rapporter og nasjonale resultater. PasOpp-rapport nr. 2 2015. Os/o: Nasjonalt kunnskapssenter for helsetjenesten 2015.

12 Jha AK, Prasopa-Plaizier N, Larizgoitia I, et al. Patient safety research: an overview of the global evidence. Qual Saf Health Care 2010;19:42-7.

13 AlHW (Australian Institute of Health and Welfare). Admitted patient care 2015-16: Australian hospital statistics. Health Services Series no. 75. Cat. no. HSE 185. Canberra: AlHW, 2017.

14 OECD (Organisation for Economic Co-operation and Development). Health at a Glance 2017: OECD indicators. Paris: OECD, 2017.

15 Rafter N, Hickey A, Conroy RM, et al. The Irish national adverse events study (INAES): the frequency and nature of adverse events in Irish hospitals-a retrospective record review study. BMJ Qual Saf 2017;26:1-9.

16 Panagioti M, Khan K, Keers RN, et al. Prevalence, severity, and nature of preventable patient harm across medical care settings: systematic review and meta-analysis. BMJ 2019;366:14185.

17 WHO. Methods and measures used in primary care patient safety research. Geneva: World Health Organization, 2008.

18 Ferrah N, Lovell JJ, Ibrahim JE. Systematic review of the prevalence of medication errors resulting in hospitalization and death of nursing home residents. J Am Geriatr Soc 2017;65:433-42.
19 Provonost PJ. Transforming patient safety: a sector-wide systems approach. Report of the WISH patient safety forum 2015. Doha: World Innovation Summit for Health (WISH), 2015.

20 Vincent C, Amalberti R. Safer healthcare: strategies for the real world. London: Springer Open, 2016.

21 Wears R, Sutcliffe K. Still not safe. Patient safety and the middlemanaging of American Medicine. New York, Oxford, 2020.

22 Nemeth CP, Herrera I. Building change: resilience engineering after ten years. Reliab Eng Syst Saf 2015;141:1-4

23 Berg SH, Akerjordet K, Ekstedt M, et al. Methodological strategies in resilient health care studies: an integrative review. Saf $\mathrm{Scl}$ 2018:110:300-12.

24 Anderson JE, Ross AJ, Jaye P. Resilience engineering in healthcare: Moving from epistemology to theory and practice. REA Symposium on Resilience Engineering, June 25-27 Soesterberg, The Netherlands 2013.

25 Iflaifel M, Lim RH, Ryan K, et al. Resilient health care: a systematic review of conceptualisations, study methods and factors that develop resilience. BMC Health Serv Res 2020;20:324.

26 Ellis LA, Churruca K, Clay-Williams R, et al. Patterns of resilience: a scoping review and bibliometric analysis of resilient health care. Saf Sci 2019;118:241-57.

27 Ross AJ, Murrells T, Kirby T, et al. An integrated statistical model of emergency department length of stay informed by resilient health care principles. Saf Sci 2019;120:129-36.

28 Lundberg J, Johansson BJE. Systemic resilience model. Reliab Eng Syst Saf 2015;141:22-32.

29 Anderson JE, Ross A, Jaye P. Modelling resilience and researching the gap between work-as-imagined and work-as-done. In: Braithwaite J, Wears RL, Hollnagel E, eds. Resilient health care. 3. CRC Press, 2019.

30 Wiig S, Fahlbruch B. Exploring Resilience: A Scientific Journey from Practice to Theory. Springer Open, Springer Briefs in Applied Sciences and Technology. Switzerland: Cham, 2019.

31 Laugaland K, Aase K. The demands imposed by a health care reform on clinical work in transitional care of the elderly: a multi-faceted Janus. In: Wears RL, Hollnagel E, Braithwaite J, eds. Resilient health care. Farnham: Ashgate, 2015: 2. 39-58.

32 Schubert CC. Patients as a source of resilience. In: Wears RL, Hollnagel E, Braithwaite J, eds. Resilient health care. Farnham: Ashgate, 2015: 2. 207-25.

33 O'Hara JK, Aase K, Waring J. Scaffolding our systems? Patients and families 'reaching in' as a source of healthcare resilience. BMJ Qual Saf 2018.

34 Bergerød IJ, Braut GS, Wiig S. Resilience from a Stakeholder perspective: the role of next of kin in cancer care. J Patient Saf 2018

35 Comfort LK, Boin A, Demchak CC. Designing resilience: preparing for extreme events. Pittsburgh: Uni Pittsburgh Press, 2010.

36 Macrae $\mathrm{C}$. Reconciling regulation and resilience in health care. In: Hollnagel E, Braithwaite J, Wears RL, eds. Resilient health care. Farnham: Ashgate, 2013: 111-22

37 Wiig S, Aase K, von Plessen C, et al. Talking about quality: exploring how 'quality' is conceptualized in European hospitals and healthcare systems. BMC Health Serv Res 2014;14.

38 Anderson J, Aase K, Bal R, et al. Exploring resilience in healthcare in six countries - an international comparative study protocol 2020 forthcoming.

39 Helsedirektoratet. Nasjonal handlingsplan for pasientsikkerhet OG kvalitetsforbedring 2019-2023. Helsedirektoratet, 2019.

40 Norwegian statistics 2019.

41 Molven O, Ferkis J. Healthcare, welfare and law. Health legislation as a mirror of the Norwegian welfare state. Oslo, NO: Gyldendal Akademisk, 2011.

42 Stein BD, Kataoka S, Jaycox LH, et al. Theoretical basis and program design of a school-based mental health intervention for traumatized immigrant children: a collaborative research partnership. J Behav Health Serv Res 2002;29:318-26.

43 Mitteness LS, Barker JC. Collaborative and team research. In: Seale C, ed. Qualitative research practice. London: SAGE Publications, 2004.

44 Svennson L, Ellström P-E, Brulin G. Introduction - on interactive research.. Int J Act Res 2007;3:233-49.

45 Langer EJ. Mindful learning. Curr Dir Psychol Sci 2000;9:220-3.

46 Weick K. Making sense of the organization. Oxford: Blackwell Publishing, 2001

47 Vale LJ, Campanella TJ. The resilient city: How modern societies recover from disaster. New York: Oxford University Press, 2005.

48 Sandelowski M, Barroso J. Handbook for Synthesizing Qualitative Research. New York: Springer Publishing Company, 2007.

49 Varvasovszky Z, Brugha R. How to do (or not to do) a stakeholder analysis. Health Pol Plan 2000;15:338-45. 
50 Elo S, Kyngäs $\mathrm{H}$. The qualitative content analysis process. $J$ Adv Nurs 2008;62:107-15.

51 Moore GF, Audrey S, Barker M, et al. Process evaluation of complex interventions: medical Research Council guidance. BMJ 2015;350:h1258.
52 Gittell JH. Rethinking autonomy: relationships as a source of resilience in a changing healthcare system. Health Serv Res 2016;51:1701-5.

53 DeWalt KM, DeWalt BR. Participant observation: a guide for fieldworkers. Walnut Creek, CA: Alta Mira Press, 2002. 